\title{
Über die Wirkung des Akonitins auf die Darmbewegung.
}

\author{
Von \\ Michinosuke Yokota. \\ (横田道之 助) \\ (Aus dem phamakologischen Institut von Prof. S. Yagi \\ an der Tohoku Universität zu Sendai.)
}

Die bisher mitgeteilten Versuche über die Wirkungsweise des Akonitins auf die Darmbewegung sind nach den mir zugänglichen Literaturangaben äusserst spärlich. $\mathrm{Pohl}^{1)}$ beobachtete beim Kaninchen, dass das Bepinseln der Darmserosa mit Akonitinlösung eine stürmische peristaltische Kontraktion hervorruft. Ganz umgekehrte Resultate boten die Versuche von Cash und Dunstan ${ }^{2)}$ dar, indem durch die Einführung des Akonitins in die Darmschlinge die Peristaltik bedeutend stark vermindert, ja sogar aufgehoben wurde. Noch Lubbe ${ }^{3 \text { ) }}$ hat es keinen nachweisbaren Einfluss auf die Darmperistaltik. Woher kommt es nun, dass die Versuchsresultate der genannten Autoren so sehr voneinander abweichen, dass sie fast in keinem Punkte im Einklang stehen? Das hat mich veranlasst, die Versuche unter verschiedenen Bedingungen anzustellen, die Wirkungsweise des Akonitins auf den Kaninchendarm festzustellen.

\section{Die Wirkung des Akonitins auf den Darm in situ.}

Bei einem kurarisierten, in Rückenlage aufgebundenen Kaninchen, bei welchem künstliche Atmung vorgenommen wurde, wurde die Bauchhöhle durch einen Schnitt entlang der Linea alba vorsichtig eröffnet. Ein kleiner Teil des blossgelegten Dünndarms wurde fixiert und mit

1) Pohl, Arch. f. exp. Pathol. u. Pharmakol., 1895, 34, 87.

2) Cash und Dunstan, Heffter's Handbuch d. exp. Pharmakol., 1920, Bd. 2, Heft 1, 301.

3) Lu bbe, Biberfeld's Ergebnisse d. exp. Toxikol., 1919, Bd. 2, 128. 
einem Schreibhebel so verbunden, dass dieser die Darmbewegung, die möglicherweise nach der Verabreichung des Mittels etwaige Veränderungen zeigen würden, auf dem Kymographion zweckmässig registriert, wodurch sich die Wirkung des Akonitins wohl leicht ersichtlich zeigen wird.

Das von mir verwendete Akonitin ist Japakonitin, welches von Herrn Prof. Majima aus japanischem Akonitum vollkommen chemisch rein dargestellt wurde und für dessen Überlassung ich ihm hier meinen besten Dank ausspreche. Bei der Verwendung wurde es zuerst zu einer salzsauren Verbindung gemacht und in Ringer' scher Flüssigkeit gelöst.

Es kommt bei dem Kaninchendarm, welcher auf eben erwähnte Weise präpariert ist, immer zu einer beträchtlichen Vergrösserung der

Fig. 1.

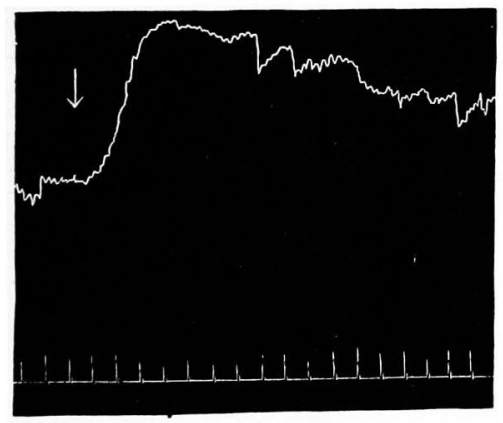

Fig. 1. Kaninchen. Kurarisiert und künstliche Atmung vorgenommen. Darm in situ. Bei dem Pfeil Aufpinseln 0,1\% iger Japakonitinlösung auf die Darmserosa. Zeitmarkierung in 15 Sekunden. Bewegung mit gesteigertem Tonus, wenn eine 0,001 bis $0,1 \%$ iger Akonitinlösung auf die Serosa des Dünndarms, wie Pohl ausführte, aufgepinselt wird. In einer stärkeren Konzentration aber tritt meist eine so heftige Kontraktion ein, dass die Pendelbewegungen mehr oder weniger verschwinden. Fig. 1 gibt ein gutes Beispiel, durch das die Veränderung der Darmbewegung beim Bepinseln mit 0,1 \%iger Lösung veranschaulicht wird. Führt man weiter bei anderen Tieren $1 \mathrm{ccm} 0,1$ o\% iger Lösung mit einer Spritze in die Darmschlinge ein, so wird die Darmbewegung angeregt und der Tonus verstärkt, wie bei den vorigen Versuchen. Darauf folgt allmähliches Absinken des Tonus bis zur Erschlaffung des Darms nach ungefähr 30 Minuten, und die Bewegung wird auch immer schwächer, um schliesslich vollständig aufzuhören. Wenn man aufs neue bei anderen Tieren 0,5 ccm 0,01 \% iger Lösung pro Kilo Körpergewicht in die Ohrvene einspritzt, so führt es sogleich zum Verschwinden des Tonus und zum völligen Stillstand der Darmbewegung, wie man es aus Fig. 2 deutlich ersieht. 
Fig. 2.

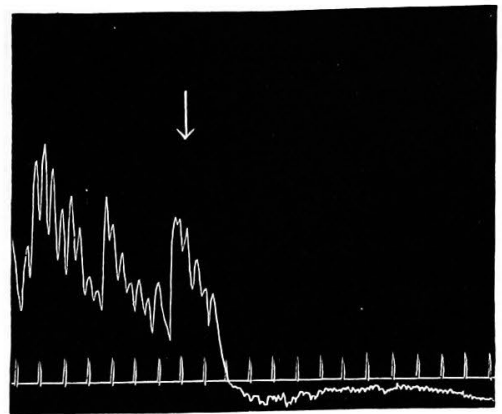

Fig. 2. Kaninchen. Kuraresiert und künstliche Atmung vorgenommen. Darm in situ. Bei dem Pfeil intravenöse Injektion von $0,00005 \mathrm{~g}$ Japakonitin. Zeitmarkierung in 15 Sekunden.
Also mit Pohl'scher Angabe stimmen die Ergebnisse meiner Versuche insofern überein, als die direkte Applikation des Akonitins auf den Darm in situ, sei sie inneroder ausserhalb der Darmschlinge vorgenommen, immer eine Verstärkung der Bewegung und eine Zunahme des 'Tonus herbeiführt. Bei intravenöser Injektion tritt eine Erschlaffung des Darms und einen Stillstand der Darmbewegung ein. Dass die Einführung des Akonitins in die

Darmschlinge im späteren Stadium diese herbeiführt, wird dadurch bedingt sein, dass diese Erscheinung, wie bei der intravenösen Injektion, wahrscheinlich durch die Resorption des Mittels ins Blut zum Vorschein kommt. Die Mitteilung von Cash und Dunstan, dass das Akonitin einen Stillstand der Darmperistaltik herbeiführt, wird doch wahrscheinlich nur auf der Beobachtung von resorptiver Wirkung beruhen.

Es kommt sogleich zu einer Tonussteigerung, wenn man die genannte Menge Akonitin an Tieren intravenös injiziert, welche wie erwähnt vorher vorbereitet und dann mit einer ausreichenden Menge Nikotin so stark vergiftet wurden, dass das Verhalten des Darms durch die Reizung der sympathischen Ganglien nicht mehr beeinflusst wird. Bei solchen Versuchen aber trat jedesmal nur die oben erwähnte Tonussteigerung, aber keine Verstärkung der Pendelbewegungen auf.

Das Nikotin hat bekanntlich eine lähmende Wirkung auf sympathische Ganglienzellen. Nach der Darreichung desselben führt die intravenöse Einspritzung des Akonitins keine Erschlaffung, sondern eine Tonuszunahme des Darms herbei. Dass es bei intravenöser Injektion des Akonitins zur Erschlaffung des Darms kommt, muss also dadurch bedingt sein, dass seine periphere d.h. tonussteigernde Wirkung auf den Darm dabei verdeckt wird, indem das Mittel die den Darm innervierenden hemmenden sympathischen Ganglienzellen reizt. Und nach der Nikotinvergiftung wird das intravenös injizierte Akonitin keine 
Verstärkung der Darmbewegung herbeiführen, da die darmbewegungbefördernde Wirkung des Akonitins infolge der Lähmung vom Auerbach'schen Plexus durch das Nikotin ausgeschaltet ist. Ich habe hier wiederum Versuche am isolierten, überlebenden Darm angestellt, um die Wirkungsweise des Akonitins auf den Darm festzustellen.

\section{Die Wirkung des Akonitins auf den isolierten, uberlebenden Darm.}

Ein kleines Stück vom Dünndarm wurde einem Kaninchen entnommen, ins Bad von $100 \mathrm{ccm}$ körperwarmer Ring er' scher Flüssigkeit

Fig. 3.

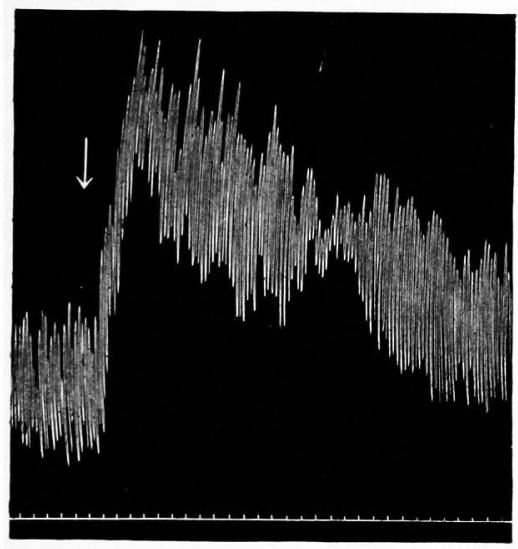

Fig. 3. Kaninchen. Isolierter überlebender Darm. Bei dem Pfeil Zusatz von $0,0003 \mathrm{~g}$ Japakonitin. Zeitmarkierung in 15 Sekunden. eingetaucht und dann die Bewegung auf dem Kymographion mit einem Schreibhebel graphisch registriert. Die Akonitinlösung wurde mit einer Spritze vorsichtig dem Bad zugesetzt und dann die Wirkung auf die Darmbewegung beobachtet. Setzt man nun $0,0001 \mathrm{~g}$ Akonitin dem $\mathrm{Bad} z u$, so tritt eine Tonussteigerung und eine Zunahme der Amplitüde ein, welche aber so vorübergehend sind, dass sie früher oder später verschwinden, so dass sich schliesslich früherer Zustand zeigt, wie Fig. 3 dieses Verhalten veranschaulicht. Auf Zusatz von über 0,001 g Akonitin erfolgt eine deutliche Tonussteigerung und zwar vielmehr eine Amplitüdeverkleinerung. Auch dieser Zustand ist nicht anhaltend, sondern er dauert eine längere oder kürzere Zeit an je nach der Grösse der Akonitindose, worauf sich allmählich eine Erschlaffung und Zunahme der Amplitüde und schliesslich früherer Zustand einstellt. Dieser Zustand ist nicht verschieden von Veränderungen, welche beim direkten Bepinseln der Darmschlinge in situ auftraten. Daraus ergibt sich, dass die Tonussteigerung und Bewegungsverstärkung, welche ich beim direkten Bepinseln der Darmschlinge 
erkannte, durch die periphere Wirkung des Akonitins herbeigeführt werden muss. Auf welche Art und Weise sind die oben beschriebenen Veränderungen durch Akonitin hervorgerufen worden?

Dass die Tonussteigerung und Bewegungsverstärkung auf Zusatz von Akonitin erfolgten, nachdem eine ausreichende Menge Atropin vorher zugesetzt war, ist fast gleich wie an dem nicht atropinisierten Darm. Diese Substanz müsste also die genannten Erscheinungen herbeigeführt haben, indem sie entweder den Auerbach'schen Plexus oder den Muskel angreift. Setzt man jedoch bei der durch Akonitin hervorgerufenen, deutlichen Tonussteigerung eine grosse Dose Atropin dem Bad zu, so tritt ein sofortiges Absinken des Tonus ein, dessen Grad deutlich ist; die Erschlaffung ist aber niemals vollständig, wenn man auch eine recht grosse Menge Atropin hinzufügt. Wenn der Tonus und der Zustand der Bewegung nach Zusatz einer grossen Menge Nikotin in früheren Zustand zurückgekommen sind, und der Zusatz von Strychnin mehr keinen Einfluss auf den Darm ausübt, so führt ein solcher von Akonitin zu einer, wenn auch geringeren Tonuszunahme und Verstärkung der Bewegung. Das Akonitin wirkt also hauptsächlich auf den Auerbach'schen Plexus oder auf den Muskel und zugleich auch auf die Vagusendigungen erregend. Infolgedessen müsste es die geschilderten Erscheinungen nach sich gezogen haben.

Da aber der Zusatz von Akonitin keine Veränderung des Zustandes des Darms mehr herbeiführt, nachdem eine grosse Dose Nikotin und Atropin zugesetzt war, so müsste sein Angriffspunkt nicht im Muskel, sondern im Auerbach'schen Pluxus liegen. Sogar hat diese Substanz keine Wirkung auf die Sympathikusendigungen. Wenn eine grosse Menge Akonitin, wie schon gesagt, auf den Darm wirkt, so hat sie eine Zeitlang eine 'Tonuszunahme und Verstärkung der Bewegung zur Folge, welche allmählich zur Norm zurückkehren. Wird dieselbe Dose aber dabei aufs nene zugesetzt, so wird die Wirkung auf den Darm schwächer als früher. Bei weiterem widerholtem Zusatz wird die Wirkung immer schwächer, um schliesslich zu verschwinden. Obgleich die Konzentration des Akonitins auf solche Weise gesteigert, $0,03 \%$ erreicht, so dauern die Bewegungen des Darmes doch selbt nach 2 Stunden rhythmisch an, welche, abgesehen nur von der Verkleinerung der Amplituide, fast nicht verschieden sind von denselben vor der Vergiftung. Daraus ergibt sich, dass diese Substanz wenigstens in geschilderten Dosen wohl nicht auf den Darm und zwar nicht auf die nervösen Apparate lähmend wirkt, weil diese auch nach wiederholter 
Darreichung von grossen Mengen Akonitin auf Nikotin, Pilokarpin und Adrenalin ebenso gut wie vorher reagieren.

\section{Zusammenfassung.}

Das Akonitin erregt für die Darmbewegungen hauptsächlich sowohl den Auerbach'schen Plexus als auch die Vagusendigungen. Wenn man also es direkt dem Darm beibringt, so führt es eine Tonuszunahme und Bewegungsverstärkung herbei. Diese Substanz hat aber eine Eigenschaft, die in den Verlauf des Sympathikus eingeschalteten Ganglienzellen zu reizen, während sie auf die Sympathikusendigungen nicht einwirkt. Wenn sie also resorbiert wird, so muss sie durch Reizung der Ganglienzellen die Darmberwegungen hemmen. Da diese Wirkung eine stärkere ist als die schon erwähnte befördernde, so kommt die Darmbewegung vielmehr nach der Resorption des Mittels zur Erschlaffung und zum Stillstand. Die bisherige Angabe, dass das Akonitin auf die Darmbewegung erregend wirkt, wird also darauf beruhen, dass bei den Versuchen nur eine unmittelbar nach der Darreichung sich einstellende Einwirkung auf den Darm beobachtet worden ist und ferner die Angabe, dass es auf die Darmbewegung hemmend wirkt, wird wohl darauf zurückzuführen sein, das nur eine erst nach der Resorption aufgetretene Erscheinung beobachtet wurde. 